\title{
Accelerated versus Standard Corneal Cross-linking for Progressive Keratoconus in Syria
}

\author{
Abdelrahman M Salman ${ }^{1}$, MD; Taym R Darwish¹, MD, PhD; Yusra H Haddad ${ }^{2}$, MD, PhD \\ Rafea H Shabaan ${ }^{3}$, MD, PhD; Mohammad Z Askar², MD, MS \\ ${ }^{1}$ Department of Ophthalmology, Tishreen University, Latakia, Syria \\ ${ }^{2}$ Department of Ophthalmology, Damascus University, Damascus, Syria \\ ${ }^{3}$ Tartous University, Faculty of Medicine, Tartous, Syria \\ ORCID: \\ Abdelrahman M Salman: https://orcid.org/0000-0002-1042-8153
}

\section{Abstract}

Purpose: To compare the outcomes of accelerated versus standard corneal cross-linking for the treatment of progressive keratoconus.

Methods: In this retrospective comparative study, 63 eyes of 40 patients with progressive keratoconus were divided into two groups; 27 eyes in group one were treated with an accelerated protocol $\left(10 \mathrm{~mW} / \mathrm{cm}^{2}, 9 \mathrm{~min}\right)$ and 36 eyes in group two were treated with the standard method $\left(3 \mathrm{~mW} / \mathrm{cm}^{2}, 30 \mathrm{~min}\right)$. Visual acuity, refraction, corneal topography, corneal tomography, and anterior and posterior corneal higher-order aberrations (HOAs) were assessed preoperatively and 18-30 months postoperatively.

Results: The LogMAR uncorrected and corrected distance visual acuity values were improved in both groups postoperatively. However, the improvement was significantly higher in group one $(P<0.05$, all). The flattening in the anterior keratometry readings, flat $\mathrm{K}$, steep $\mathrm{K}$, and average $\mathrm{K}$ were significantly higher in group two $(P<0.001$, all). The maximum anterior keratometry (AKf) values significantly decreased in both groups, whereas the maximum posterior keratometry (AKb) values increased. The reduction in the minimum corneal thickness (ThKmin) was significantly greater (36.49um) in group two, compared to 10.85 um in group one. There was a significant increase in the posterior average keratometry, and a significant decrease in the posterior astigmatism, along 3 $\mathrm{mm}$ meridian in S-CXL $(P=0.03, P=0.008$, respectively), while the corresponding values showed no statistical significance in group one $(P>0.05)$. The anterior corneal trefoil was significantly reduced in group one $(P=0.002)$, whereas anterior total HOAs and coma were significantly improved in group two $(P<0.0014$, all). The posterior corneal spherical aberration decreased significantly in group one $(P=0.02)$, while group two revealed significant reduction in the posterior trefoil values $(P=0.011)$. The change in the anterior maximum keratometry was significantly and positively correlated to the preoperative maximum keratometry in group two $(P=0.53, P=0.003)$.

Conclusion: An accelerated cross-linking protocol using $10 \mathrm{~mW} / \mathrm{cm}^{2}$ for 9 min showed more visual improvement and less pachymetric reduction when compared to the standard protocol, however, anterior corneal flattening, posterior corneal steepening, and the change in the posterior astigmatism were significantly higher in the standard protocol; while corneal HOAs were improved in both protocols.

Keywords: Accelerated; Corneal Crosslinking; HOAs; Keratometry; Posterior Astigmatism; Standard 


\section{INTRODUCTION}

Corneal cross-linking was first introduced by Wollensask et al in 2003. ${ }^{[1]}$ Zhang et al demonstrated that the term "collagen crosslinking" is misleading, as the actual cross-links, induced by interaction of ultraviolet A (UVA) and riboflavin, occurs between the amino terminals of the collagen side chains and the proteoglycans of the extracellular matrix and not between and within the collagen fibers. ${ }^{[2]}$ The standard corneal cross-linking (S-CXL) reported by Wollensask et al depends on using $3 \mathrm{~mW} / \mathrm{cm}^{2}$ fluence for $30 \mathrm{~min}$ to achieve a total irradiance of $5.4 \mathrm{~J} / \mathrm{cm}^{2}{ }^{[1]}$ More recently, a variety of CXL protocols - in term of fluency, time, epithelial integrity, and different indications for $C X L$ - have been introduced. ${ }^{[3-5]}$ The accelerated corneal cross-linking (A-CXL) uses high energy (up to $30 \mathrm{~mW} / \mathrm{cm}^{2}$ ) for shorter time (3-10 min). Bunsen-Rosoe law of reciprocity states that an increased intensity coupled with reduced exposure time theoretically delivers a total dose to the tissue equivalent to that applied in standard treatment. ${ }^{[6]}$ Evaluations of the difference of the outcomes between $\mathrm{S}-\mathrm{CXL}$ and $\mathrm{A}-\mathrm{CXL}$ and their impact on the anterior corneal flattening, hyperopic shift, astigmatism, and corneal thinning have gained a particular importance for the refractive surgeons in choosing the CXL protocol to combine with other refractive surgery procedure. ${ }^{[7-9]}$

In this study, we aimed to compare the visual outcomes, topographic parameters, and corneal higher-order aberrations (HOAs) values (anterior and posterior) of an A-CXL $10 \mathrm{~mW} / \mathrm{cm}^{2}, 9 \mathrm{~min}$ and the standard CXL protocol using $3 \mathrm{~mW} / \mathrm{cm}^{2}, 30 \mathrm{~min}$.

\section{METHODS}

This study was approved by the Research Ethics Committee of Tishreen University in accordance

\section{Correspondence to:}

Abdelrahman M Salman, MD. Honorary Clinical Lecturer at Tishreen University, Scientific Director of Tartous Specialist Eye Center, Tartous, Syria P.O. Box: 25.

E-mail: abd.r.salman10@gmail.com

Received: 18-07-2020 Accepted: 29-04-2021

\section{Access this article online}

Website: https://knepublishing.com/index.php/JOVR

DOI: 10.18502/jovr.v16i3.9430 with the tenets of the Declaration of Helsinki. Informed consent, in Arabic language, was obtained from all patients over 18 years or their guardians if under 18 at the time of cross-linking. A retrospective, comparative study was performed at the Department of Ophthalmology, Tishreen University Hospital, Syria. All patients who underwent epithelium-off corneal cross-linking between January 2016 and February 2017 were recruited. Sixty-three eyes of 40 patients were included in this analysis. Patients in whom bilateral cross-linking was performed and underwent different types of procedure ( $A-C X L$ vs $S-C X L$ ) were included to avoid inter-eye correlation. Subjects were divided into two groups: 27 eyes in group one were treated with A-CXL and 36 eyes of group two underwent S-CXL. Diagnosis of keratoconus was made if (a) there was irregular cornea determined by distorted keratometry mires or distortion of the dilated retinoscopic reflex (or combination of these) in addition to (b) at least two of the following topographic/tomographic findings: abnormal posterior ectasia, abnormal thickness distribution, or symmetry index front (SIf) > $1.17 \mathrm{D}$; or one of the following slit-lamp findings: Vogt striae, 2-mm arc of Fleisher ring, or corneal scaring consistent with keratoconus. ${ }^{[10]}$ Progression of keratoconus was defined as: at least 1 diopter increase in the anterior maximum keratometry (AKf) or in the manifest refraction spherical equivalent (MRSE), decrease of $5 \%$ in the minimum pachymetry, or loss of at least two lines of the corrected distance visual acuity (CDVA) during the past 12 months. Patients under 18 years were cross-linked without waiting for progression. ${ }^{[1]}$ Patients who had preoperative pachymetry < $400 \mu \mathrm{m}$, previous ocular surgery, corneal scar, pregnancy, lactation, herpetic keratitis, or dry eye were excluded. All patients had comprehensive ocular examination, including the measurement of uncorrected distance visual acuity (UDVA) and CDVA, manifest refraction, slit lamp examination, and funduscopy. Topographic and tomographic measurement, as well as corneal total HOAs,

This is an open access journal, and articles are distributed under the terms of the Creative Commons Attribution-NonCommercial-ShareAlike 4.0 License, which allows others to remix, tweak, and build upon the work non-commercially, as long as appropriate credit is given and the new creations are licensed under the identical terms.

How to cite this article: Salman AM, Darwish TR, Haddad YH, Shabaan $\mathrm{RH}$, Askar MZ. Accelerated versus Standard Corneal Cross-linking for Progressive Keratoconus in Syria. J Ophthalmic Vis Res 2021;16:338-348. 
coma, trefoil, and spherical aberrations (anterior and posterior at $6 \mathrm{~mm}$ optical zone) were obtained by Placido Scheimpflug-tomographer, Sirius (CSO, Italy). These investigations were carried out in all patients preoperatively and 18-30 months postoperatively. Visual acuity was converted to LogMAR units. All patients were instructed to discontinue the use of hard or soft contact lenses, for at least three and one weeks, respectively, prior to their examination and CXL.

\section{Surgical Technique}

The "epi-off" CXL technique was used in both groups. Topical proparacaine hydrochloride 0.5\% (Proparacaine Rama, Rama Pharma, Syria) anesthetic eye drops were administrated every 3 min starting 10-15 min before surgery. The central corneal epithelium (8-9 $\mathrm{mm}$ ) was removed using blunt spatula and dry sponge, without alcohol assistance. Riboflavin with dextran $(0.1 \%$ riboflavin in $20 \%$ dextran, Medicross, Germany) solution was instilled every $3 \mathrm{~min}$ for $60 \mathrm{~min}$, starting $30 \mathrm{~min}$ before irradiance and continuing for 30 min during the irradiance, in S-CXL group. In A-CXL group, riboflavin was instilled every 2 min for $29 \mathrm{~min}$, starting 20 min before irradiance and continuing for 9 min during the UVA irradiance. The irradiance was commenced after saturation of the anterior chamber with riboflavin. This was inspected by slit-lamp examination as fluorescence within the anterior chamber. In S-CXL group, eyes were treated with UV-X (Peschke Meditrade Gmbh, Hueneberg, Switzerland) system; $3 \mathrm{~mW} / \mathrm{cm}^{2}$ was applied for $30 \mathrm{~min}$ to achieve the total energy of $5.4 \mathrm{~J} / \mathrm{cm}^{2}$. Eyes of the A-CXL group were irradiated with the Vega C.B.M-X Linker (CSO, Italy) using the A-CXL $10 \mathrm{~mW} / \mathrm{cm}^{2}$ for 9 min to reach the same total energy. After UVA irradiance, the corneal surface was irrigated with balanced salt solution and soft contact lens was applied for 3-4 days. Topical moxifloxacin 0.5\% (Megamox, Rama Pharma, Syria) eye drop was prescribed four times daily for one week and topical fluorometholone 0.1\% (Methouflor 0.1\%, Diamond Pharma, Syria) eye drop was applied four times daily for two weeks, which was then tapered to twice daily for two weeks.

\section{Statistical Analysis}

Statistical Package for Social Sciences Software version 20 (SPSS, INC, Chicago, IL, USA) was applied. Data were expressed as mean \pm standard deviation (SD). Two samples independent $T$-test and paired $T$-test were applied for normally distributed variables, while non-parametric test was used if a paired $T$-test was applied to compare the postoperative with the baseline outcomes. $P$ value $<0.05$ was considered significant.

\section{RESULTS}

\section{Baseline Characteristics}

Sixty-three eyes of 40 patients were included in this study. Of these, 27 eyes of 15 patients (9 females, 18 males, mean age; $23.13 \pm 7.72$ years) underwent A-CXL and 36 eyes of 25 patients (24 females, 12 males, mean age; $23.4 \pm 7.37$ years) underwent S-CXL. There was no significant difference between the two groups in terms of demographic, UDVA, CDVA, MRSE, topography, pachymetry, and corneal HOAs except for anterior trefoil values, $0.94 \pm 48 \mu \mathrm{m}$ in $\mathrm{A}-\mathrm{CXL}$ group versus $0.64 \pm 0.41 \mu \mathrm{m}$ in $\mathrm{S}-\mathrm{CXL}(P=0.011)$. Females were significantly higher in A-CXL group $(P=0.009)$ [Tables 1 and 4].

\section{Visual, Refractive, and Anterior Keratometry Outcomes}

The UDVA and CDVA values were significantly improved postoperatively compared with baseline in A-CXL group $(P<0.05)$. In S-CXL, the UDVA and CDVA values were improved but the improvement did not reach statistical significance $(P>0.05)$. In terms of mean MRSE, there was a nonsignificant myopic shift in group one, and significant hyperopic shift in group two (A-CXL: $0.28 \mathrm{D}, P=0.65$; $\mathrm{S}-\mathrm{CXL}$ : $-0.47 \mathrm{D}, P=0.05)$. Both groups had slight reduction in the Sim cylinder values postoperatively (A-CXL: $-0.14 \mathrm{D}, P=0.39$, S-CXL: $-0.22 \mathrm{D}, P=0.08)$. K1, $\mathrm{K} 2$, and Avg $\mathrm{K}$ did not show significant change in A-CXL group (0.19 D, -0.11 D, $-0.04 \mathrm{D}$, respectively) $(P \geq 0.2$, all), while the corresponding values were significantly decreased in S-CXL group (K1: 1.47 $\mathrm{D}, \mathrm{K} 2: 1.21 \mathrm{D}$, Avg K: $1.34 \mathrm{D}, P<0.001$, all). AKf (maximum anterior keratometry) values were significantly reduced in both groups (A-CXL; $0.8 \mathrm{D}$, $P=0.03$, S-CXL; $1.93 \mathrm{D}, P=0.001$ ) [Table 2].

The change in Snellen CDVA in both groups was as follows: in the A-CXL group, 39.13\% and in the S-CXL group, $29.41 \%$ gained one 
Table 1. Patient characteristics at baseline

Treatment type

\begin{tabular}{|c|c|c|c|c|c|}
\hline & & Tech 9 min & Tech $30 \mathrm{~min}$ & & \\
\hline \multicolumn{6}{|l|}{$N(\%)$} \\
\hline No. of subjects & & $15(37.5)$ & $25(62.5)$ & & \\
\hline \multirow[t]{2}{*}{ No. of eyes } & & $27(42.86)$ & 36 (57.14) & & \\
\hline & & & & Total & $P$-value \\
\hline \multirow[t]{2}{*}{ Sex, $n$ (\%) } & M & $18(66.67)$ & $12(33.33)$ & 30 (47.62) & 0.009 \\
\hline & $\mathrm{F}$ & 9 (33.33) & 24 (67.67) & 33 (52.38) & \\
\hline \multirow[t]{2}{*}{ Eye, $n$ (\%) } & OD & $16(59.26)$ & 19 (52.78) & 35 (55.56) & 0.608 \\
\hline & OS & $11(40.74)$ & $17(47.22)$ & $28(44.44)$ & \\
\hline Age, mean $\pm S D$ & & $23.13 \pm 7.72$ & $23.4 \pm 7.37$ & $23.3 \pm 7.4$ & 0.9138 \\
\hline
\end{tabular}

$\mathrm{P}$, paired test; Values in bold are significant $(P<0.05)$

line postoperatively. The corresponding values were $4.35 \%$ and $14.71 \%$ regarding the gain of two or more lines, respectively. In the $A-C X L$ group, $8.7 \%$ and in the S-CXL group, $5.88 \%$ lost one line. The corresponding figures were $0.0 \%$ and $17.65 \%$ in terms of the loss of two or more lines, respectively. Totally, $47.83 \%$ of eyes in the A-CXL group and $32.35 \%$ in the $\mathrm{S}-\mathrm{CXL}$ group displayed no change [Figure 1].

\section{Pachymetry, Posterior Corneal Keratometry, and Astigmatism Outcomes}

The minimum corneal thickness was reduced $10.85 \mu \mathrm{m}$ in the A-CXL group and $36.49 \mu \mathrm{m}$ in the S-CXL group $(P=0.029$ and $P=0.002$, respectively). The baseline values of $A K b$ (the steepest point of the posterior surface) increased from $77.08 \mathrm{D}$ to $79.25 \mathrm{D}$ in the $\mathrm{A}-\mathrm{CXL}$ group, and from $78.08 \mathrm{D}$ to $80.08 \mathrm{D}$ in the $\mathrm{S}-\mathrm{CXL}$ group. The average posterior keratometry Avg $\mathrm{K}$ (bck) along 3- and 5-mm back meridians were not significantly changed in the $\mathrm{A}-\mathrm{CXL}$ group $(P>0.9$, all), while they significantly increased (representing posterior steepening) in the S-CXL group $(P<0.05$, all). The posterior corneal astigmatism (PCA) did not significantly change along the corresponding meridians in the $A-C X L$ group ( $P>0.5$, all). In the S-CXL group, PCA values decreased (absolute values increased) significantly in the 3 - and 5-mm meridians $(P<0.009$, all) [Table 3].

\section{Anterior and Posterior Corneal Higher-order Aberrations Outcomes}

In the $A-C X L$ group, the anterior trefoil was significantly decreased $(P=0.0024)$, whereas total HOAs, coma, and spherical aberrations showed no statistically significant difference $(P>0.05$, all). The anterior total HOAs and coma aberrations were significantly improved in the $\mathrm{S}-\mathrm{CXL}$ group $(P=0.0008$ and $P=0.001$, respectively), while the trefoil and the spherical aberrations revealed nonsignificant change $(P>0.02$, all). There was a significant reduction in the posterior spherical aberration values $(P=0.0027)$ in the A-CXL group; while the posterior total HOAs, trefoil, and coma did not significantly change $(P>0.01$, all). The total HOAs, coma, and spherical aberrations were not significantly changed in the S-CXL group $(P>$ 0.014 , all), while the trefoil significantly decreased $(P=0.014)$ [Table 4].

\section{Correlation Analysis Outcomes}

Both groups revealed no significant correlation between the preoperative measurements (UDVA, CDVA, and ThKMin) and the change in AKf values at the follow-up. However, the change in AKf was significantly and positively correlated to the preoperative AKf in group two $(\mathrm{R}=0.53, P=0.003)$ [Table 5].

The mean difference for each parameter from baseline and final follow-up time were compared in both groups. The changes were not statistically significant between the two groups, except for 
Table 2. Visual, refractive, and anterior keratometric outcomes

\begin{tabular}{|c|c|c|c|c|c|c|}
\hline & & \multicolumn{2}{|c|}{ Tech $9 \min (n=27)$} & \multicolumn{2}{|c|}{ Tech $30 \min (n=36)$} & \multirow[b]{2}{*}{$P$-value } \\
\hline & & Mean & Std. Dev. & Mean & Std. Dev. & \\
\hline \multirow[t]{4}{*}{ UDVA (LogMAR) } & Preoperative & 0.66 & 0.46 & 0.57 & 0.37 & 0.3433 \\
\hline & Postoperative & 0.52 & 0.41 & 0.50 & 0.36 & 0.8176 \\
\hline & Mean change & 0.14 & 0.20 & 0.07 & 0.26 & 0.2825 \\
\hline & $\mathrm{P}^{*}$ value & 0.0029 & & 0.11 & & \\
\hline \multirow[t]{4}{*}{ CDVA (LogMAR) } & Preoperative & 0.32 & 0.27 & 0.32 & 0.23 & 0.8333 \\
\hline & Postoperative & 0.27 & 0.21 & 0.29 & 0.23 & 0.7371 \\
\hline & Mean change & 0.06 & 0.11 & 0.03 & 0.20 & 0.5167 \\
\hline & $\mathrm{P}^{*}$ value & 0.03 & & 0.37 & & \\
\hline \multirow[t]{4}{*}{ MRSE (D) } & Preoperative & -2.49 & 2.89 & -1.76 & 1.04 & 0.1623 \\
\hline & Postoperative & -2.78 & 3.65 & -1.29 & 1.71 & 0.042 \\
\hline & Mean change & 0.28 & 2.99 & -0.47 & 1.33 & 0.2004 \\
\hline & $\mathrm{P}^{*}$ value & 0.65 & & 0.05 & & \\
\hline \multirow[t]{4}{*}{ Topo Cyl (D) } & Preoperative & -3.24 & 1.81 & -2.83 & 1.39 & 0.2531 \\
\hline & Postoperative & -3.10 & 1.39 & -2.61 & 1.25 & 0.1716 \\
\hline & Mean change & -0.14 & 0.77 & -0.22 & 0.71 & 0.695 \\
\hline & $\mathrm{P}^{*}$ value & 0.39 & & 0.08 & & \\
\hline \multirow[t]{4}{*}{$\mathrm{K} 1$ (D) } & Preoperative & 44.91 & 0.34 & 45.50 & 3.08 & 0.3498 \\
\hline & Postoperative & 44.73 & 0.35 & 44.03 & 2.57 & 0.2426 \\
\hline & Mean change & 0.19 & 0.14 & 1.47 & 1.54 & 0.0001 \\
\hline & $\mathrm{P}^{*}$ value & 0.20 & & $<0.0001$ & & \\
\hline \multirow[t]{4}{*}{ K2 (D) } & Preoperative & 48.44 & 2.38 & 48.21 & 3.12 & 0.8272 \\
\hline & Postoperative & 48.55 & 3.06 & 47.00 & 2.77 & 0.0431 \\
\hline & Mean change & -0.11 & 1.39 & 1.21 & 1.20 & 0.0002 \\
\hline & $\mathrm{P}^{*}$ value & 0.69 & & $<0.0001$ & & \\
\hline \multirow[t]{4}{*}{ Avg K (D) } & Preoperative & 46.83 & 2.08 & 46.80 & 3.01 & 0.9575 \\
\hline & Postoperative & 46.86 & 2.61 & 45.46 & 2.55 & 0.0403 \\
\hline & Mean change & -0.04 & 1.99 & 1.34 & 1.31 & 0.0019 \\
\hline & $\mathrm{P}^{*}$ value & 0.9211 & & $<0.0001$ & & \\
\hline \multirow[t]{4}{*}{$A K f(D)$} & Preoperative & 55.04 & 4.09 & 54.78 & 3.84 & 0.7238 \\
\hline & Postoperative & 54.24 & 4.61 & 52.84 & 3.31 & 0.0531 \\
\hline & Mean change & 0.80 & 1.39 & 1.93 & 2.73 & 0.1205 \\
\hline & $\mathrm{P}^{*}$ value & 0.0312 & & 0.0011 & & \\
\hline
\end{tabular}

UDVA, uncorrected distance visual acuity; CDVA, corrected distance visual acuity; MRSE, manifest refraction spherical equivalent; Cyl, sim cylinder value; K1, flat keratometry; K2, steep keratometry; AvgK, anterior average keratometry; AKf, apical keratoscopy front; $\mathrm{D}$, diopter

P, Paired test; $\mathrm{P}^{*}$, student's test; Values in bold are significant $(P<0.05)$

ThKmin, Sim keratometry (K1, K2, KAvg), KAvg (bck, 5), anterior total HOAs, trefoil and coma, and posterior total HOAs and trefoil $(P<0.05$, for all) [Tables 2-4]. 
Table 3. Pachymetry, posterior corneal keratometries, and astigmatism outcomes

\begin{tabular}{|c|c|c|c|c|c|c|}
\hline & & \multicolumn{2}{|c|}{ Tech $9 \min (n=27)$} & \multicolumn{2}{|c|}{ Tech $30 \min (n=36)$} & \multirow[b]{2}{*}{$P$-value } \\
\hline & & Mean & Std. Dev. & Mean & Std. Dev. & \\
\hline \multirow[t]{4}{*}{ ThkMin $(\mu \mathrm{m})$} & Preoperative & 436.54 & 26.51 & 449.46 & 33.81 & 0.1062 \\
\hline & Postoperative & 425.69 & 27.55 & 412.97 & 47.23 & 0.2252 \\
\hline & Mean change & 10.85 & 22.05 & 36.49 & 51.07 & 0.0198 \\
\hline & $\mathrm{P}^{*}$ value & 0.02 & & 0.0002 & & \\
\hline \multirow[t]{4}{*}{$\mathrm{AKb}(\mathrm{D})$} & Preoperative & 77.08 & 7.69 & 78.69 & 8.74 & 0.9647 \\
\hline & Postoperative & 79.25 & 8.33 & 81.08 & 9.56 & 0.6893 \\
\hline & Mean change & -2.17 & 4.01 & -2.39 & 9.73 & 0.93 \\
\hline & $\mathrm{P}^{*}$ value & 0.0406 & & 0.2315 & & \\
\hline \multirow[t]{4}{*}{ AvgK (bck, 3) (D) } & Preoperative & -7.22 & 0.70 & -7.03 & 0.86 & 0.5818 \\
\hline & Postoperative & -7.20 & 0.94 & -6.66 & 1.29 & 0.0731 \\
\hline & Mean change & -0.02 & 0.66 & -0.36 & 0.97 & 0.1276 \\
\hline & $\mathrm{P}^{*}$ value & 0.9131 & & 0.0386 & & \\
\hline \multirow[t]{4}{*}{ AvgK (bck, 5) (D) } & Preoperative & -6.97 & 0.48 & -6.88 & 0.74 & 0.787 \\
\hline & Postoperative & -6.97 & 0.61 & -6.59 & 0.93 & 0.0637 \\
\hline & Mean change & 0.00 & 0.42 & -0.29 & 0.60 & 0.0436 \\
\hline & $\mathrm{P}^{*}$ value & 0.9609 & & 0.011 & & \\
\hline \multirow[t]{4}{*}{ Cyl (back, 3) (D) } & Preoperative & -0.92 & 1.20 & -1.09 & 0.53 & 0.3278 \\
\hline & Postoperative & -1.36 & 0.74 & -2.19 & 2.12 & 0.1088 \\
\hline & Mean change & 0.44 & 1.25 & 1.10 & 2.18 & 0.186 \\
\hline & $\mathrm{P}^{*}$ value & 0.1045 & & 0.0086 & & \\
\hline \multirow[t]{4}{*}{ Cyl (back, 5) (D) } & Preoperative & -0.71 & 0.82 & -0.88 & 0.55 & 0.7552 \\
\hline & Postoperative & -1.21 & 1.22 & -1.79 & 2.11 & 0.2665 \\
\hline & Mean change & 0.50 & 1.32 & 0.91 & 1.85 & 0.3643 \\
\hline & $\mathrm{P}^{*}$ value & 0.0819 & & 0.0092 & & \\
\hline
\end{tabular}

ThiKMin, minimum corneal thickness; AKb, apical kertoscopy back; AvgK (bck, 3), average keratometry along 3 mm back meridian; AvgK (bck, 5), average keratometry along 5 mm back meridian; Cyl (bck, 3), cylinder value along 3 mm back meridian; Cyl (bck, 5), cylinder value along $5 \mathrm{~mm}$ back meridian; D, diopter

$\mathrm{P}$, Paired test; $\mathrm{P}^{*}$, student's test; Values in bold are significant $(P<0.05)$

\section{DISCUSSION}

Several studies have revealed that both A$C X L$ and $S-C X L$ are effective in halting the progression of keratoconus. ${ }^{[12,13]}$ Tomita et al published the first article comparing the standard and accelerated protocols. ${ }^{[14]}$ They reported no significant difference in the mean UDVA and CDVA, keratometric readings, or the postoperative MRSE values. In contrast, we found improvement in the logMAR visual acuity in both groups. The UDVA and CDVA significantly improved $(0.14, P$ $=0.002$ and $0.06, P=0.03$, respectively) in the
A-CXL group, compared to $0.07, P=0.11$ and $0.03, P$ $=0.37$, respectively, in the S-CXL group. There was no statistically significant difference between the two groups. However, 12 eyes of the A-CXL group lost two lines of the Snellen DCVA at three month postoperatively. The reduction in visual acuity at this stage was attributed to the increase in the corneal HOAs and the decrease in the contrast sensitivity. Ghanavati et al stated that increased corneal HOAs and decreased contrast sensitivity were the factors responsible for deceased visual acuity at the early postoperative period after crosslinking. ${ }^{[15]}$ Fortunately, none of the eyes treated 
Table 4. Anterior and posterior corneal HOAS outcomes

\begin{tabular}{|c|c|c|c|c|c|c|}
\hline & & \multicolumn{2}{|c|}{ Tech $9 \min (n=27)$} & \multicolumn{2}{|c|}{ Tech $30 \min (n=36)$} & \multirow[b]{2}{*}{$P$-value } \\
\hline & & Mean & Std. Dev. & Mean & Std. Dev. & \\
\hline \multicolumn{7}{|l|}{ Anterior corneal HOAs 6 mm } \\
\hline \multirow[t]{4}{*}{ Anterior total HOAS (RMS, $\mu \mathrm{m})$} & Preoperative & 2.35 & 1.27 & 2.62 & 1.30 & 0.4248 \\
\hline & Postoperative & 2.25 & 1.27 & 2.16 & 1.13 & 0.5737 \\
\hline & Mean change & 0.10 & 0.33 & 0.46 & 0.73 & 0.0269 \\
\hline & $\mathrm{P}^{*}$ value & 0.1329 & & 0.0008 & & \\
\hline \multirow[t]{4}{*}{ Trefoil (RMS, $\mu \mathrm{m})$} & Preoperative & 0.94 & 0.48 & 0.64 & 0.41 & 0.0115 \\
\hline & Postoperative & 0.82 & 0.50 & 0.72 & 0.40 & 0.399 \\
\hline & Mean change & 0.12 & 0.18 & -0.08 & 0.42 & 0.0269 \\
\hline & $\mathrm{P}^{*}$ value & 0.0024 & & 0.2688 & & \\
\hline \multirow[t]{4}{*}{ Coma (RMS, $\mu \mathrm{m})$} & Preoperative & 2.04 & 1.17 & 2.26 & 1.31 & 0.5091 \\
\hline & Postoperative & 1.94 & 1.32 & 1.82 & 1.13 & 0.5074 \\
\hline & Mean change & 0.10 & 0.41 & 0.44 & 0.74 & 0.0433 \\
\hline & $\mathrm{P}^{*}$ value & 0.2323 & & 0.0013 & & \\
\hline \multirow[t]{4}{*}{ Spherical aberration (RMS, $\mu \mathrm{m}$ ) } & Preoperative & 0.11 & 0.37 & 0.05 & 0.45 & 0.5833 \\
\hline & Postoperative & 0.08 & 0.34 & 0.01 & 0.38 & 0.3639 \\
\hline & Mean change & 0.04 & 0.15 & 0.04 & 0.31 & 0.9096 \\
\hline & $\mathrm{P}^{*}$ value & 0.2567 & & 0.4272 & & \\
\hline \multicolumn{7}{|l|}{ Posterior corneal HOAs 6 mm } \\
\hline \multirow[t]{4}{*}{ Posterior total HOAS (RMS, $\mu \mathrm{m}$ ) } & Preoperative & 0.86 & 0.49 & 1.19 & 1.00 & 0.0923 \\
\hline & Postoperative & 0.93 & 0.50 & 0.90 & 0.57 & 0.8215 \\
\hline & Mean change & -0.07 & 0.29 & 0.28 & 0.84 & 0.037 \\
\hline & $\mathrm{P}^{*}$ value & 0.1912 & & 0.0636 & & \\
\hline \multirow[t]{4}{*}{ Trefoil (RMS, $\mu \mathrm{m})$} & Preoperative & 0.56 & 0.43 & 0.69 & 0.61 & 0.3604 \\
\hline & Postoperative & 0.62 & 0.44 & 0.46 & 0.33 & 0.106 \\
\hline & Mean change & -0.05 & 0.27 & 0.23 & 0.52 & 0.0115 \\
\hline & $\mathrm{P}^{*}$ value & 0.2944 & & 0.0146 & & \\
\hline \multirow[t]{4}{*}{ Coma (RMS, $\mu \mathrm{m})$} & Preoperative & 0.38 & 0.21 & 0.54 & 0.45 & 0.052 \\
\hline & Postoperative & 0.38 & 0.20 & 0.44 & 0.26 & 0.3425 \\
\hline & Mean change & 0.00 & 0.12 & 0.10 & 0.40 & 0.2003 \\
\hline & $\mathrm{P}^{*}$ value & 0.8433 & & 0.1594 & & \\
\hline \multirow[t]{4}{*}{ Spherical aberration (RMS, $\mu \mathrm{m})$} & Preoperative & -0.05 & 0.09 & -0.14 & 0.27 & 0.209 \\
\hline & Postoperative & -0.08 & 0.07 & -0.14 & 0.22 & 0.106 \\
\hline & Mean change & 0.03 & 0.07 & 0.00 & 0.24 & 0.519 \\
\hline & $\mathrm{P}^{*}$ value & 0.027 & & 0.9096 & & \\
\hline
\end{tabular}

HOAs, higher order aberrations; RMS, root mean square

$\mathrm{P}$, Paired test; $\mathrm{P}^{*}$, student's test; Values in bold are significant $(P<0.05)$ 
Table 5. Correlation between AKf Change at follow-up and preoperative assessment variables

\begin{tabular}{lccccc}
\hline AKf change & & Preop UDVA & Preop CDVA & Preop ThiKMin & Preop AKf \\
\hline Tech 9 min & $\mathrm{R}$ & 0.3855 & 0.1228 & -0.4321 & -0.2302 \\
& $P$-value & 0.1265 & 0.6387 & 0.0832 & 0.374 \\
Tech $30 \mathrm{~min}$ & $\mathrm{R}$ & -0.0207 & 0.0845 & -0.0711 & 0.5365 \\
& $P$-value & 0.9182 & 0.6751 & 0.7246 & $\mathbf{0 . 0 0 3 9}$ \\
\hline
\end{tabular}

UDVA, uncorrected distance visual acuity; CDVA, corrected distance visual acuity; ThiKMin, minimum corneal thickness; AKf, apical keratoscopy front

P, Student's test; Values in bold are significant $(P<0.05)$; R, correlation coefficient

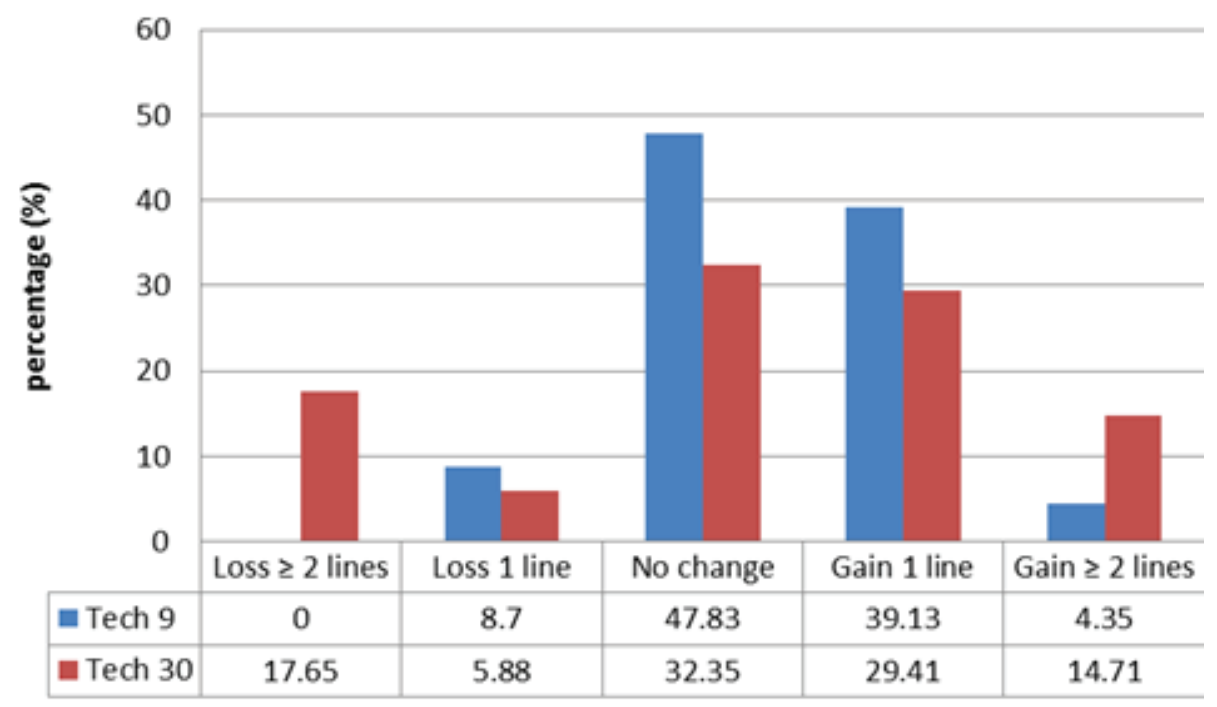

Figure 1. corrected distance visual acuity changes after 9 and 30 min corneal cross-linking (Snellen).

with A-CXL lost two or more lines of Snellen CDVA at the final follow-up. However, this was not the case for the eyes treated with S-CXL, as six eyes lost two or more lines at the final examination. This could be explained by the significant persistent haze formation in four eyes and scar development in two eyes. Our study showed a slight myopic shift of the MRSE in the A-CXL group and significant hyperopic shift $(O .47 \mathrm{D})$ in the S-CXL group, while Sim cylinder values were nonsignificantly reduced in both groups. Hashemi et al reported a significant reduction in keratometric readings in the $\mathrm{S}-\mathrm{CXL}$ treated eyes but not in the A-CXL treated ones and concluded that the flattening effect was higher in the S-CXL group. ${ }^{[16]}$ This is in agreement with our results. We found significant flattening in the mean flat, steep, and average keratometry values (1.47 D, 1.20 D, $1.99 \mathrm{D}$, respectively) with the S-CXL protocol, while the corresponding values did not show any statistically significantly deference in the
A-CXL group. The anterior maximum keratometry or $\mathrm{K}$ max, which is considered as the most sensitive indicator for KC progression, ${ }^{[17,18]}$ was significantly reduced after treatment in both $\mathrm{A}-\mathrm{CXL}$ and $\mathrm{S}-\mathrm{CXL}$ groups $(P<0.05$, all). The mean ThKmin values decreased $10.85 \mu \mathrm{m}$ in the $\mathrm{A}-\mathrm{CXL}$ group compared to $36.49 \mu \mathrm{m}$ in the S-CXL group. Shetty et al observed that the minimum thickness reduction was higher in the S-CXL group. ${ }^{[19]}$ Greenstein et al suggested that the decrease in thickness was related to an increased compactness of the cross-linked cornea. ${ }^{[20]}$ However, the marked reduction in corneal thickness after S-CXL in our study may represent a measurement artefact. Dependence on Scheimpfug-based pachymetric measurements was one of our study limitations. Anterior segment optical coherence tomography (AS-OCT) showed higher repeatability compared with Scheimpflug imaging devices in measuring the corneal thickness. ${ }^{[21]}$ 
Patients with KC are more likely to develop cataract at a younger age than normal subjects ${ }^{[22]}$ and many of them will eventually require cataract surgery and toric intraocular lens implantation. Koch et $\mathrm{al}^{[23]}$ reported that neglecting PCA which is higher in $\mathrm{KC}$ patients compared to normal population ${ }^{[24]}$ will lead to the overcorrection in eyes having with the rule astigmatism and the undercorrection in eyes with the against the rule astigmatism. Safarzadeh et al found a nonsignificant difference in PCA and a significant increase in the mean posterior maximum keratometry values after S-CXL treatment. ${ }^{[25]}$ In contrast, we evaluated the changes of posterior astigmatism and posterior keratometry along the 3- and 5-mm meridians between the baseline and the postoperative follow-up in both groups. The Cyl (bck) values decreased (absolute values increased) nonsignificantly along with the studied meridians in the $\mathrm{A}-\mathrm{CXL}$ group. While the decrease was significant in the S-CXL group. The difference in Cyl (bck) values along the 3-mm meridian was 0.44 $\mathrm{D}$ in the $\mathrm{A}-\mathrm{CXL}(P=0.1)$ and $1.10 \mathrm{D}$ in the S-CXL $(P=$ $0.0086)$ groups. The mean posterior keratometry AvgK (bck3) increased (absolute values decreased) nonsignificantly in the $A-C X L$ group, while the increase was significant in the $\mathrm{S}-\mathrm{CXL}$ group along the 3-mm meridian (0.02 D, $P=0.91$; 0.36 $\mathrm{D}, \quad P=0.038$, respectively). AKb values (the steepest point of the posterior surface) increased postoperatively, $2.17 \mathrm{D}$ in the $\mathrm{A}-\mathrm{CXL}$ group and 2.39 $\mathrm{D}$ in the S-CXL group. We hypothesize the difference in PCA outcomes between our study and the study by Safarzadeh et al can be attributed to the difference in the type of the topographer used, as Safarzadeh depended on Pentacam measurement. Although Pentacam and Sirius are both Scheimpfug-based tomographers, Sirius showed good to excellent repeatability ${ }^{[26-28]}$ and was less affected than Pentacam by the post-CXL haze. ${ }^{[2]}$ The increase in both the posterior mean and maximum keratometries represent increased posterior corneal steepening. Twa et al evaluated the corneal changes after 5,211 myopic LASIK procedures. They suggested that the posterior steepening is a response to the anterior flattening induced by myopic LASIK correction. ${ }^{[30]}$ Kirgiz et al considered posterior corneal steepening as important as anterior corneal flattening for stabilizing the keratometric values and enhancing the visual acuity. ${ }^{[31]}$
Recent studies have revealed increased spherical and coma aberrations in eyes with KC compared to normal population. ${ }^{[32]}$ Greenstein et al reported significant improvement in the anterior corneal HOAs and coma after S-CXL treatment. ${ }^{[33]}$ However, they found no statistically significant difference in the posterior corneal HOAs. This is to some extent in agreement with our findings; anterior total HOAs and coma were significantly reduced $(P<0.0014$, all), while posterior corneal trefoil was significantly deceased in the S-CXL group. Ozulken et al found significant difference in coma aberrations after $10 \mathrm{~min}$ at $9 \mathrm{~mW} / \mathrm{cm}^{2}$ UVA irradiance. ${ }^{[34]}$ In our study, anterior trefoil and posterior spherical aberrations values were significantly improved in the A-CXL group $(P=0.002$ and $P=0.02$, respectively). Ghanem et al concluded that the improvement in HOAs in KC patients is attributed to the flattening of the corneal apex caused by the CXL effect. ${ }^{[35]}$

To the best of our knowledge, this the first study to compare the impact of different CXL protocols on the PCA. Lack of demarcation line depth measurements, low number of patients, and the retrospective design of the study were limiting factors in this study. Larger cohort studies to evaluate the effect of CXL on the orientation of the astigmatism and the correlation between the anterior and posterior astigmatism changes are needed.

In summary, we found that S-CXL resulted in significantly higher anterior corneal flattening, more increase in posterior steepening, further decrease in posterior astigmatism, and more reduction in the minimum thickness than the accelerated-CXL. However, both protocols showed improvement in the postoperative visual acuity and the corneal HOAs, but the improvement in the visual acuity was significantly higher in the $A-C X L$ protocol.

\section{Financial Support and Sponsorship}

Nil.

\section{Conflicts of Interest}

There are no conflicts of interest. 


\section{REFERENCES}

1. Wollensak G, Spoerl E, Seiler T. Riboflavin/ultravioleta-induced collagen crosslinking for the treatment of keratoconus. Am J Ophthalmol 2003;135:620-627.

2. Zhang $\mathrm{Y}$, Conrad $\mathrm{AH}$, Conrad GW. Effects of ultraviolet$A$ and riboflavin on the interaction of collagen and proteoglycans during corneal cross-linking. J Biol Chem 2011;286:13011-13022.

3. Kirgiz A, Eliacik M, Yildirim Y. Different accelerated corneal collagen cross-linking treatment modalities in progressive keratoconus. Eye Vis 2019;6:16.

4. Saleem MIH, Ibrahim Elzembely HA, AboZaid MA, Elagouz M, Saeed AM, Mohammed OA, et al. Three-year outcomes of cross-linking plus (combined cross-linking with femtosecond laser intracorneal ring segments implantation) for management of keratoconus. $J$ Ophthalmol 2018;2018:6907573.

5. Cifariello F, Minicucci M, Di Renzo F, Di Taranto D, Coclite G, Zaccaria S, et al. Epi-off versus epi-on corneal collagen cross-linking in keratoconus patients: a comparative study through 2-year follow-up. J Ophthalmol 2018;2018:4947983.

6. Bunsen RW, Roscoe HE. Photochemical researches - part $\mathrm{V}$. On the measurement of the chemical action of direct and diffuse sunlight. Proc $R$ Soc Lond 1863;12:306-312.

7. Woo JH, lyer JV, Lim L, Hla MH, Mehta JS, Chan CMI, et al. Conventional versus accelerated collagen cross-linking for keratoconus: a comparison of visual, refractive, topographic and biomechanical outcomes. Open Ophthalmol J 2017;11:262-272.

8. Aldahlawi NH, Hayes S, O’Brart DP, Meek KM. Standard versus accelerated riboflavin-ultraviolet corneal collagen crosslinking: resistance against enzymatic digestion. J Cataract Refract Surg 2015;41:1989-1996.

9. Zhu AY, Jun AS, Soiberman US. Combined protocols for corneal collagen cross-linking with photorefractive surgery for refractive management of keratoconus: update on techniques and review of literature. Ophthalmol Ther 2019;8:15-31.

10. Arbelaez MC, Versaci F, Vestri G, Barboni P, Savini G. Use of a support vector machine for keratoconus and subclinical keratoconus detection by topographic and tomographic data. Ophthalmology 2012;119:2231-2238.

11. Reeves SW, Stinnett S, Adelman RA, Afshari NA. Risk factors for progression to penetrating keratoplasty in patients with keratoconus. $A m \mathrm{~J}$ Ophthalmol 2005;140:607-611.

12. Nicula C, Pop R, Rednik A, Nicula D. 10-year results of standard cross-linking in patients with progressive keratoconus in Romania. J Ophthalmol 2019;2019:8285649.

13. Bozkurt E, Ozgurhan EB, Akcay BI, Kurt T, Yildirim Y, Günaydin ZK, et al. Refractive, topographic, and aberrometric results at 2-year follow-up for accelerated corneal cross-link for progressive keratoconus. J Ophthalmol 2017;2017:5714372.

14. Tomita M, Mita M, Huseynova T. Accelerated versus conventional corneal collagen crosslinking. J Cataract Refract Surg 2014;40:1013-1020.

15. Zarei-Ghanavati $\mathrm{S}$, Khakshour $\mathrm{H}$, Vejdani $\mathrm{M}$, Ghooshkhanei $H$, Vejdani A. Evaluation of changes in visual acuity, contrast sensitivity and aberrations in patients with keratoconus after corneal collagen cross-linking. J Ophthalmic Vis Res 2017;12:260-264.

16. Hashemi H, Fotouhi A, Miraftab M, Bahrmandy H, Seyedian MA, Amanzadeh $\mathrm{K}$, et al. Short-term comparison of accelerated and standard methods of corneal collagen crosslinking. J Cataract Refract Surg 2015;41:533-540.

17. Wittig-Silva $C$, Whiting $M$, Lamoureux $E$, Lindsay RG, Sullivan LJ, Snibson GR. A randomized controlled trial of corneal collagen cross-linking in progressive keratoconus: preliminary results. J Refract Surg 2008;24:S720-S725.

18. Sykakis E, Karim R, Evans JR, Bunce C, Amissah-Arthur $\mathrm{KN}$, Patwary $\mathrm{S}$, et al. Corneal collagen cross-linking for treating keratoconus. Cochrane Database Syst Rev 2015;3:CD010621.

19. Shetty R, Pahuja NK, Nuijts RM, Ajani A, Jayadev C, Sharma $C$, et al. Current protocols of corneal collagen crosslinking: visual, refractive, and tomographic outcomes. Am J Ophthalmol 2015;160:243-249.

20. Greenstein SA, Shah VP, Fry KL, Hersh PS. Corneal thickness changes after corneal collagen crosslinking for keratoconus and corneal ectasia: one-year results. $J$ Cataract Refract Surg 2011;37:691-700.

21. Wongchaisuwat N, Metheetrairat A, Chonpimai P, Nujoi W, Prabhasawat P. Comparison of central corneal thickness measurements in corneal edema using ultrasound pachymetry, Visante anterior-segment optical coherence tomography, Cirrus optical coherence tomography, and Pentacam Scheimpflug camera tomography. Clin Ophthalmol 2018;12:1865-1873.

22. Thebpatiphat N, Hammersmith KM, Rapuano CJ, Ayres $\mathrm{BD}$, Cohen EJ. Cataract surgery in keratoconus. Eye Contact Lens 2007;33:244-246.

23. Koch DD, Jenkins RB, Weikert MP, Yeu E, Wang L. Correcting astigmatism with toric intraocular lenses: effect of posterior corneal astigmatism. J Cataract Refract Surg 2013;39:1803-1809.

24. Aslani F, Khorrami-Nejad M, Aghazadeh Amiri M, Hashemian H, Askarizadeh F, Khosravi B. Characteristics of posterior corneal astigmatism in different stages of keratoconus. J Ophthalmic Vis Res 2018;13:3-9.

25. Safarzadeh M, Nasiri N, Doostdar A, Kamali M. Comparative study of changes of corneal curvatures and uncorrected distance visual acuity prior to and after corneal collagen crosslinking: 1 -year results. Taiwan $J$ Ophthalmol 2016;6:127-130.

26. Finis D, Ralla B, Karbe M, Borrelli M, Schrader S, Geerling G. Comparison of two different scheimpflug devices in the detection of keratoconus, regular astigmatism, and healthy corneas. J Ophthalmol 2015;2015:315281.

27. Lanza M, Paolillo E, Gironi Carnevale UA, Lanza A, Irregolare $\mathrm{C}$, Mele $\mathrm{L}$, et al. Central corneal thickness evaluation in healthy eyes with three different optical devices. Cont Lens Anterior Eye 2015;38:409-413.

28. Kumar M, Shetty R, Jayadev C, Rao HL, Dutta D. Repeatability and agreement of five imaging systems for measuring anterior segment parameters in healthy eyes. Indian J Ophthalmol 2017;65:288-294.

29. Shetty R, Agrawal A, Deshmukh R, Kaweri L, Rao HL, Nagaraja $H$, et al. Effect of post crosslinking haze on the repeatability of Scheimpflug-based and slit-scanning 
imaging devices. Indian J Ophthalmo/ 2017;65:305-310.

30. Twa MD, Roberts C, Mahmoud AM, Chang JS Jr. Response of the posterior corneal surface to laser in situ keratomileusis for myopia. J Cataract Refract Surg 2005;31:61-71.

31. Kırgız A, Atalay K, Çabuk KŞ, Kaldırım H, Tașkapılı M. Factors affecting visual acuity after accelerated crosslinking in patients with progressive keratoconus. Arq Bras Oftalmol 2016;79:151-154.

32. Alió JL, Shabayek MH. Corneal higher order aberrations: a method to grade keratoconus. J Refract Surg 2006;22:539-545.
33. Greenstein SA, Fry KL, Hersh MJ, Hersh PS. Higherorder aberrations after corneal collagen crosslinking for keratoconus and corneal ectasia. J Cataract Refract Surg 2012;38:292-302.

34. Özülken K, Aksoy Aydemir G, Aydemir E, Kızıltoprak $H$, Yüksel E. Comparison of two different accelerated corneal cross-linking procedure outcomes in patients with keratoconus. Balkan Med J 2020;37:131-137.

35. Ghanem RC, Santhiago MR, Berti T, Netto MV, Ghanem VC. Topographic, corneal wavefront, and refractive outcomes 2 years after collagen crosslinking for progressive keratoconus. Cornea 2014;33:43-48. 